\title{
Synthesis of robust gain scheduling based fault detection filters for a class of parameter uncertain nonlinear systems
}

\author{
Andras Varga
}

\begin{abstract}
We discuss the solution of the fault detection problem for parameter uncertain nonlinear systems using a gain scheduling based approach. By using a set of linearized plant models, a set of least order linear fault detection filters is determined to serve for interpolation based gain scheduling. The basic synthesis approach is the nullspace method for constant linear systems, whose extension to the case of linear parameter varying (LPV) models provides the methodological framework for the synthesis of robust fault detection filters. The resulting gain scheduled fault detection filter provides robustness with respect to both model nonlinearities as well as parametric uncertainties. In a general setting, we consider the case when part of the uncertain parameters are non-measurable and part of them are measurable.
\end{abstract}

\section{INTRODUCTION}

Addressing robustness aspects in the synthesis of residual generators for fault detection is a active area of present day research. For robust controller synthesis, the use of gain scheduling techniques represents a practical way to cope with uncertainties originating from plant nonlinearities and parametric variations. Various features of controller gain scheduling techniques are described in the ample survey [1]. A main aspect which justifies the present work is the fact that gain scheduling allows the application of powerful linear design tools to solve difficult synthesis problems for nonlinear systems operating in the neighborhood of some equilibrium points. In the last few years, several numerically reliable synthesis methods of residual generators have been developed and implemented as robust numerical software [2]. The availability of such tools potentially allows the application of gain scheduling techniques in solving robust fault detection problems for nonlinear systems as well.

There are basically two ways to arrive to gain scheduled fault detection filters for nonlinear systems. The first way involves obtaining a family of linearized plant models to be used to build linear parameter varying (LPV) models to approximate the underlying nonlinear model. Such models can be obtained in several way, see for example [3], [4], but for most practical applications, the generation effort is considerable. Using the generated LPV models, special methods can be employed to obtain parameter dependent fault detector filters. For example, geometric synthesis methods for affine LPV-models have been proposed in [5] and applied to fault detection filter synthesis in [6]. This approach is primarily intended for robust fault detection using LPV gain scheduling schemes in the case when all uncertain parameters

A. Varga is with German Aerospace Center, DLR-Oberpfaffenhofen Institute of Robotics and Mechatronics, D-82234 Wessling, Germany andreas.varga@dlr.de are measurable, and is less suited to address the robust fault detection in the presence of parametric uncertainties.

Recently, an alternative approach to address robust fault detection problems has been proposed in [7], where the general case is considered when a part of uncertain parameters is measurable and thus can be used for an LPV gain scheduling, and another part is non measurable, for which robustness must be enforced. This procedure extends the nullspace approach of [8] and [9] to LPV models and provides a way to achieve via LPV gain scheduling robustness simultaneously with respect to measurable and nonmeasurable uncertain parameters. Symbolic linear algebra tools for rational nullspace computation and numerical robust optimization techniques for worst-case parameter fitting are required to perform the proposed synthesis approach. The applicability of this approach crucially depends on the complexity of the original symbolic LPV-models. Therefore, models with complex polynomial or rational expressions may lead to unsolvable nullspace computation problems due to excessive computational times.

In this paper we follow the second (the traditional) way to obtain gain scheduling based fault detection filters, namely, using a family of linearized plant models to determine a family of least order linear fault detection filters and perform gain scheduling either by interpolating suitably the state space matrices of these filters or determining approximated LPV-representations, similarly as in the case of LPV plant modeling. Following this way, we propose an alternative synthesis approach of LPV fault detectors, which completely eliminates the need of building LPV plant models as basis for the synthesis of LPV-model based residual generators. By exploiting the existing parametric freedom in the detector synthesis problem, a particular LPV statespace representation of the least order detectors is used, with two constant and two parameter varying matrices. To obtain a continuous mapping from uncertain parameters to such a detector representation, a specialized version of the nullspace synthesis procedure of [9] is employed. To interpolate the resulting detectors, besides standard techniques (see [1] and references cited therein), a special parameter fitting technique is employed relying on a frequency-weighted formulation of the approximation problem. The proposed method is illustrated on an example.

\section{LINEAR PARAMETER-VARYING DESCRIPTIONS}

Consider the nonlinear plant model

$$
\begin{aligned}
\dot{\tilde{x}}(t) & =F(\tilde{x}, \tilde{u}, d, f, \pi) \\
\tilde{y}(t) & =G(\tilde{x}, \tilde{u}, d, f, \pi)
\end{aligned}
$$


where $\tilde{x}$ is the state vector, $\tilde{u}$ is the control input vector, $\tilde{y}$ denotes the measured output, $d$ is an unknown disturbance vector, $f$ is a fault signal vector, and $\pi$ is a constant parameter vector. The vector functions $F$ and $G$ are assumed to be differentiable with respect to all intervening variables, thus guaranteeing the existence of solution of (1) as well as of the various Jacobian matrices.

Let $\left(\tilde{x}_{0}, \tilde{u}_{0}, \tilde{y}_{0}\right)$ be a plant equilibrium point corresponding to a fixed value of $\pi$ such that

$$
\begin{aligned}
& 0=F\left(\tilde{x}_{0}, \tilde{u}_{0}, 0,0, \pi\right) \\
& \tilde{y}_{0}=G\left(\tilde{x}_{0}, \tilde{u}_{0}, 0,0, \pi\right)
\end{aligned}
$$

In a linearization based gain scheduling approach, the first step is to obtain a linear approximation of the plant around an equilibrium point, where the (measurable) scheduling variables, denoted by $\rho_{2}$, explicitly appear. In general, $\rho_{2}$ may include components of the measured output $\tilde{y}_{0}$ or quantities depending on $\tilde{y}_{0}$, as well as measurable parameters from $\pi$. We denote by $\rho_{1}$ the non-measurable components of $\pi$, and we denote by $\rho$ the vector with stacked components $\rho_{1}$ and $\rho_{2}$. To make explicit the dependence of the equilibrium point on $\rho$, we will denote it by $\left(\tilde{x}_{0}(\rho), \tilde{u}_{0}(\rho), \tilde{y}_{0}(\rho)\right)$.

Corresponding to the above equilibrium point, there is an LPV plant model of the form

$$
\begin{aligned}
& \dot{x}(t)=A(\rho) x(t)+B_{u}(\rho) u(t)+B_{d}(\rho) d(t)+B_{f}(\rho) f(t) \\
& y(t)=C(\rho) x(t)+D_{u}(\rho) u(t)+D_{d}(\rho) d(t)+D_{f}(\rho) f(t)
\end{aligned}
$$

which describes the local behavior of the nonlinear plant around the equilibrium. In (3), $x=\tilde{x}-\tilde{x}_{0}(\rho), u=\tilde{u}-\tilde{u}_{0}(\rho)$, and $y=\tilde{y}-\tilde{y}_{0}(\rho)$, and, for example,

$$
\begin{aligned}
A(\rho) & =\frac{\partial F}{\partial \tilde{x}}\left(\tilde{x}_{0}(\rho), \tilde{u}_{0}(\rho), 0,0, \pi\right), \\
B_{f}(\rho) & =\frac{\partial F}{\partial f}\left(\tilde{x}_{0}(\rho), \tilde{u}_{0}(\rho), 0,0, \pi\right), \\
D_{d}(\rho) & =\frac{\partial G}{\partial d}\left(\tilde{x}_{0}(\rho), \tilde{u}_{0}(\rho), 0,0, \pi\right) .
\end{aligned}
$$

In what follows, we assume that $x(t)$ is the $n$-dimensional system state vector, $y(t)$ is the $p$-dimensional system output vector, $u(t)$ is the $m_{u}$-dimensional control input vector, $d(t)$ is the $m_{d}$-dimensional disturbance vector, and $f(t)$ is the $m_{f}$-dimensional fault vector $f(t)$.

The vector $\rho$ is assumed to belong to a bounded region $\Pi \subset \mathcal{P}$ of the $n_{\rho}$-dimensional parameter space $\mathcal{P}$. In a more general setting we can also allow time-varying parameters $\rho(t)$. However, to simplify the notations, the dependence on time of $\rho$ will not be explicitly emphasized and we consider only slowly varying parameters which can be assimilated with constant values over sufficiently large time periods.

\section{THE ROBUST FAULT DETECTION PROBLEM}

To ease the presentation, we will assume that the parameters in $\rho$ are constant. Thus, we can alternatively use an input-output representation of the form

$$
\mathbf{y}(s)=G_{u}(s, \rho) \mathbf{u}(s)+G_{d}(s, \rho) \mathbf{d}(s)+G_{f}(s, \rho) \mathbf{f}(s),
$$

where $\mathbf{y}(s), \mathbf{u}(s), \mathbf{d}(s)$, and $\mathbf{f}(s)$ are the Laplacetransformed vectors $y(t), u(t), d(t)$, and $f(t)$, respectively, and $G_{u}(s, \rho), G_{d}(s, \rho)$, and $G_{f}(s, \rho)$ are the parameter dependent transfer-function matrices (TFMs) from the control inputs to outputs, disturbance inputs to outputs, and fault inputs to outputs, respectively. These TFM are given by

$$
\begin{aligned}
& G_{u}(s, \rho)=C(\rho)(s I-A(\rho))^{-1} B_{u}(\rho)+D_{u}(\rho) \\
& G_{d}(s, \rho)=C(\rho)(s I-A(\rho))^{-1} B_{d}(\rho)+D_{d}(\rho) \\
& G_{f}(s, \rho)=C(\rho)(s I-A(\rho))^{-1} B_{f}(\rho)+D_{f}(\rho)
\end{aligned}
$$

As already mentioned, the unknown parameter vector $\rho$ has two components: $\rho_{1} \in \Pi_{1}$, which is not measurable, and $\rho_{2} \in \Pi_{2}$, which is measurable, and $\Pi=\Pi_{1} \times \Pi_{2}$. The synthesis problem formulated bellow attempts to solve basically a robust fault detection problem with respect to $\rho_{1}$, while taking advantage of the availability of $\rho_{2}$ by attempting to achieve robustness using a gain scheduling approach.

A linear residual generator (or fault detection filter) processes the measurable system outputs $y(t)$ and control inputs $u(t)$ and generates the residual signals $r(t)$ which serve for decision making on the presence or absence of faults. We use a parameter dependent filter of the form

$$
\mathbf{r}(s)=Q\left(s, \rho_{2}\right)\left[\begin{array}{l}
\mathbf{y}(s) \\
\mathbf{u}(s)
\end{array}\right]
$$

where $Q\left(s, \rho_{2}\right)$ is the TFM of the filter, which explicitly depends on the measurable parameter $\rho_{2}$ (e.g., via an equivalent LPV state-space realization of the filter). For a physically realizable filter, $Q\left(s, \rho_{2}\right)$ must be proper with respect to $s$ (i.e., only with finite poles) and robustly stable (i.e., only with poles having negative real parts for all values of $\rho_{2}$ ). The dimension $q$ of the residual vector $r(t)$ depends on the fault detection problem to be solved. The simpler case when only $\rho_{1}$ is present leads to a constant detector $Q(s)$, while in the absence of $\rho_{1}$ only the LPV gain scheduling aspect is addressed.

The residual signal $r(t)$ in (5) generally depends via the system outputs $y(t)$ of all system inputs $u(t), d(t)$ and $f(t)$. The residual generation system, obtained by replacing in (5) $\mathbf{y}(s)$ by its expression in (4), is given by

$$
\mathbf{r}(s)=R_{u}(s, \rho) \mathbf{u}(s)+R_{d}(s, \rho) \mathbf{d}(s)+R_{f}(s, \rho) \mathbf{f}(s)
$$

where

$$
\left[R_{u}(s, \rho)\left|R_{d}(s, \rho)\right| R_{f}(s, \rho)\right]:=Q\left(s, \rho_{2}\right) G_{e}(s, \rho)
$$

with

$$
G_{e}(s, \rho):=\left[\begin{array}{c|c|c}
G_{u}(s, \rho) & G_{d}(s, \rho) & G_{f}(s, \rho) \\
I_{m_{u}} & 0 & 0
\end{array}\right]
$$

For a successfully designed filter $Q\left(s, \rho_{2}\right)$, the corresponding residual generation system is proper with respect to variable $s$, robustly stable and achieves specific fault detection requirements (e.g., exact or approximate decoupling of control and disturbance inputs from the residuals).

We can now formulate the following Robust Fault Detection Problem (RFDP): For the LPV-system (3), determine a proper and stable linear residual generator (or fault detection 
filter) having the form (5) such that for all $\rho \in \Pi$ and a given $\gamma>0$ there exists $\beta>0$ such that

(i) $\quad\|r(t)\| \leq \gamma \max \{\|u(t)\|,\|d(t)\|\}$ when $f(t)=0$ and for all $u(t), d(t)$;

(ii) $\quad\|r(t)\| \geq \beta\left\|f_{i}\right\|$ for $i=1, \ldots, m_{f}$ and for $u(t)=0, d(t)=0$;

(iii) $\quad r(t)$ is asymptotically bounded.

Here $\|\cdot\|$ denotes any usual signal norm. The gap defined as $\beta / \gamma$ measures the sensitivity of detection task, where larger values guarantees the detection of smaller faults. The exact solution of the RFDP corresponds to the case when we can choose $\gamma=0$, and the corresponding gap is $\infty$.

Besides the above requirements it is often desirable for practical use that the $q \times\left(p+m_{u}\right)$ TFM of the detector $Q\left(s, \rho_{2}\right)$ has the least possible McMillan degree. Note that as fault detector, we can always choose $Q\left(s, \rho_{2}\right)$ as a rational row vector, thus with $q=1$ (scalar) output.

The fulfillment of requirement $(i i)$ ensures that all faults produce non-zero residual responses. When designing fault detectors, this requirement for fault detectability is usually replaced by the stronger condition that persistent (e.g., constant) faults produce asymptotically persistent residuals. This requirement is known as strong fault detectability.

The requirements $(i)$ and $(i i)$ can be easily transcribed into equivalent synthesis conditions. The approximate decoupling condition $(i)$ requires that both $R_{u}(s, \rho)$ and $R_{d}(s, \rho)$ have small norms $\forall \rho$, and thus can be achieved by minimizing the worst-case norm

$$
\max _{\rho \in \Pi}\left\|\left[R_{u}(s, \rho) R_{d}(s, \rho)\right]\right\|
$$

With a suitable scaling of the detector, we can always achieve that the minimal worst-case norm is equal to a given $\gamma$.

The (detectability) condition $(i i)$ requires basically that for all $\rho$, all columns $R_{f_{i}}(s, \rho), i=1, \ldots, m_{f}$ of $R_{f}(s, \rho)$ are nonzero. Thus, $(i i)$ is fulfilled by determining $\beta$ as the maximum of

$$
\min _{i=1, \ldots, m_{f}} \min _{\rho \in \Pi}\left\|R_{f_{i}}(s, \rho)\right\|>0
$$

Additionally, the condition ( $\mathrm{iii}$ ) on the boundedness of the residual signal requires that $R_{f}(s, \rho), R_{u}(s, \rho)$, and $R_{d}(s, \rho)$ are stable TFMs for all $\rho$. In the case when the $\rho_{1}$ component is present, a necessary condition to fulfill ( $i i i)$ is the robust stability of the closed-loop plant for (4) [10]. In what follows, we will tacitly assume that this condition is fulfilled.

In [7], a synthesis procedure to solve the formulated RFDP is proposed using a combination of symbolic and numerical computational tools. In this paper, we propose an alternative approach which exclusively relies on reliable numerical tools for nominal synthesis used in conjunction with optimizationbased robustness analysis techniques.

\section{APPROXIMATE NULLSPACE METHOD}

Assume temporarily that $\rho$ is measurable. We will assume throughout this paper that the exact solvability conditions of the RPDP are fulfilled for all $\rho \in \Pi$ [11]:

$$
\operatorname{rank}\left[G_{d}(s, \rho) G_{f_{i}}(s, \rho)\right]>\operatorname{rank} G_{d}(s, \rho), i=1, \ldots, m_{f}
$$

Let $Q(s, \rho)$ be a detector which exactly solves the RFDP. Thus, $Q(s, \rho)$ is stable and proper and for all $\rho \in \Pi$ satisfies the decoupling conditions $R_{u}(s, \rho)=0$ and $R_{d}(s, \rho)=0$, or equivalently

$$
Q(s, \rho) G(s, \rho)=0,
$$

where

$$
G(s, \rho)=\left[\begin{array}{cc}
G_{u}(s, \rho) & G_{d}(s, \rho) \\
I_{m_{u}} & 0
\end{array}\right]
$$

as well as the detectability conditions

$$
R_{f_{i}}(s, \rho) \neq 0, \quad i=1, \ldots, m_{f} .
$$

From (11) it appears that $Q(s, \rho)$ is a left annihilator of $G(s, \rho)$. The nullspace synthesis method proposed in [7], determines $Q(s, \rho)$ symbolically to serve for obtaining an approximation $\bar{Q}\left(s, \rho_{2}\right)$ by minimizing, for example, the worst-case error norm

$$
\max _{\rho \in \Pi}\left\|Q(s, \rho)-\bar{Q}\left(s, \rho_{2}\right)\right\|
$$

such that the condition (9) for $Q(s, \rho)=\bar{Q}\left(s, \rho_{2}\right)$ jointly with the stability requirement on $R_{f}(s, \rho), R_{u}(s, \rho)$ and $R_{d}(s, \rho)$ are fulfilled. Possible system norms to be employed in (14) are the $\mathcal{H}_{2^{-}}, \mathcal{H}_{\infty^{-}}$, or even the $\nu$-gap norm [12]. Alternatively, the weighted worst-case error norm

$$
\max _{\rho \in \Pi}\left\|\left(Q(s, \rho)-\bar{Q}\left(s, \rho_{2}\right)\right) G_{e}(s, \rho)\right\|
$$

can be minimized to simultaneously achieve

$\bar{Q}\left(s, \rho_{2}\right) G(s, \rho) \approx 0,\left(Q(s, \rho)-\bar{Q}\left(s, \rho_{2}\right)\right)\left[\begin{array}{c}G_{f}(s, \rho) \\ 0\end{array}\right] \approx 0$ and thus to fulfill both conditions (8) and (9).

The result of the nullspace computation based approach is a parametric state-space realization for $Q(s, \rho)$ of the form

$$
Q(s, \rho)=\left[\begin{array}{l|l}
A_{Q} & B_{Q}(\rho) \\
\hline C_{Q} & D_{Q}(\rho)
\end{array}\right],
$$

where $A_{Q}$ and $C_{Q}$ are constant matrices, and only $B_{Q}(\rho)$ and $D_{Q}(\rho)$ depend on $\rho$. In what follows we describe two approaches to directly determine $\bar{Q}\left(s, \rho_{2}\right)$ in an LPVparametric form

$$
\bar{Q}\left(s, \rho_{2}\right)=\left[\begin{array}{c|c}
A_{Q} & \bar{B}_{Q}\left(\rho_{2}\right) \\
\hline C_{Q} & \bar{D}_{Q}\left(\rho_{2}\right)
\end{array}\right]
$$

where $A_{Q}$ and $C_{Q}$ are known (e.g., from a nominal design) and only the parametric matrices $\bar{B}_{Q}\left(\rho_{2}\right)$ and $\bar{D}_{Q}\left(\rho_{2}\right)$ have to be determined.

\section{A. Multi-model approach}

The traditional gain scheduling approach determines for a family of $N$ linearized models corresponding to a discrete set of points $\Pi_{N}=\left\{\rho^{(1)}, \rho^{(2)}, \ldots, \rho^{(N)}\right\}$ (obtained, for example, by parameter griding), a family of $N$ detectors $Q\left(s, \rho^{(i)}\right)$, for $i=1, \ldots, N$. We assume that all detectors share the same $A_{Q}$ and $C_{Q}$ matrices as in (16), a condition which can be easily fulfilled for scalar output detectors by using a specialized version of the synthesis method of 
[9]. Having the grid values $B_{Q}\left(\rho^{(i)}\right)$ and $D_{Q}\left(\rho^{(i)}\right)$ for $i=1, \ldots, N$, interpolation techniques can be employed to determine $B_{Q}(\rho)$ and $D_{Q}(\rho)$ for an arbitrary value of $\rho \in \Pi$. If $\rho_{1}$ is not present, then this family of detectors can be used to implement a gain scheduling based detector using multilinear interpolation (table look-up) techniques. Alternatively, parametric approximations of $\bar{B}_{Q}(\rho)$ and $\bar{D}_{Q}(\rho)$ can be determined along the line of the techniques developed in [3], [4].

In the case when $\rho_{1}$ is not empty, then $\bar{B}_{Q}\left(\rho_{2}\right)$ and $\bar{D}_{Q}\left(\rho_{2}\right)$ must be determined to robustly approximate $B_{Q}(\rho)$ and $D_{Q}(\rho)$ on $\Pi_{N}$. This can be done in several ways. If we assume that $\Pi_{N}=\Pi_{N_{1}} \times \Pi_{N_{2}}$ (i.e., $N=N_{1} N_{2}$ ), then for each $\rho_{2}^{(i)}$ value there are $N_{1}$ values of $\rho_{1}^{(j)}, j=1, \ldots, N_{1}$ and also of $B_{Q}\left(\rho_{1}^{(j)}, \rho_{2}^{(i)}\right)$ to be approximated by a single value $\bar{B}_{Q}\left(\rho_{2}^{(i)}\right)$. This value can be simply determined as the mean value of $N_{1}$ matrices, or $\bar{B}_{Q}\left(\rho_{2}^{(i)}\right)$ and $\bar{D}_{Q}\left(\rho_{2}^{(i)}\right)$ can be simultaneously determined via optimization by minimizing for each $\rho_{2}^{(i)}, i=1, \ldots, N_{2}$

$$
\max _{\rho_{1} \in \Pi_{N_{1}}}\left\|\left(Q\left(s, \rho_{1}, \rho_{2}^{(i)}\right)-\bar{Q}\left(s, \rho_{2}^{(i)}\right)\right) G_{e}\left(s, \rho_{1}, \rho_{2}^{(i)}\right)\right\|
$$

The resulting $\bar{B}_{Q}\left(\rho_{2}^{(i)}\right)$ and $\bar{D}_{Q}\left(\rho_{2}^{(i)}\right)$ for $i=1, \ldots, N_{2}$, can serve for an interpolation based gain scheduling or for building parametric approximations (see below).

To directly construct parametric approximations, we can assume for $\bar{B}_{Q}\left(\rho_{2}\right)$ a certain parametric form $\widetilde{B}_{Q}\left(\rho_{2}, \theta\right)$ (e.g., affine, polynomial, rational, etc.), where $\theta$ includes the free parameters (e.g., coefficient matrices) to be fitted by solving

$$
\bar{\theta}=\arg \min _{\theta} \max _{\rho \in \Pi_{N}}\left\|B_{Q}(\rho)-\widetilde{B}_{Q}\left(\rho_{2}, \theta\right)\right\|
$$

Then define $\bar{B}_{Q}\left(\rho_{2}\right):=\widetilde{B}_{Q}\left(\rho_{2}, \bar{\theta}\right)$ (and similarly $\bar{D}_{Q}\left(\rho_{2}\right)$ ). Alternatively, the minimization of the input-output error

$$
\bar{\theta}=\arg \min _{\theta} \max _{\rho \in \Pi_{N}}\left\|\left(Q(s, \rho)-\bar{Q}\left(s, \rho_{2}\right)\right) G_{e}(s, \rho)\right\|
$$

can be employed.

The main advantage of the multi-model approach is that for each parameter value $\rho^{(i)}$, the computation of $G_{e}\left(s, \rho^{(i)}\right)$ and the corresponding detector $Q\left(s, \rho^{(i)}\right)$ is necessary to be performed only once. This involves the trimming and linearization of the nonlinear model (1) for each parameter value $\rho^{(i)}$ and the synthesis of a detector $Q\left(s, \rho^{(i)}\right)$ using standard nullspace methods [9], [13] for which numerical software is available in the FAULT DETECTION Toolbox [2].

For the validation of the LPV detector approximation, it is necessary to perform a worst-case analysis for the resulting detector on the continuum of values $\rho \in \Pi$ by evaluating, for example, (15). A global optimization-based worst-case search can be performed for this purpose using $\|(Q(s, \rho)-$ $\left.\bar{Q}\left(s, \rho_{2}\right)\right) G_{e}(s, \rho) \|$ as criterion (to be maximized). Each function evaluation involves performing "on the fly" the chain of computations: trimming, linearization and detector synthesis.

\section{B. Continuous parameter fitting}

By using a specialized version of the synthesis method of [9], we can achieve a continuous mapping $\rho \mapsto Q(s, \rho)$ by designing detectors "on the fly". Thus, assuming a certain parametric form for the matrices $\widetilde{B}_{Q}\left(\rho_{2}, \theta\right)$ and $\widetilde{D}_{Q}\left(\rho_{2}, \theta\right)$, we can minimize (15) to determine the best fitting $\bar{\theta}$ and the corresponding $\bar{B}_{Q}\left(\rho_{2}\right):=\widetilde{B}_{Q}\left(\rho_{2}, \bar{\theta}\right)$ and $\bar{D}_{Q}\left(\rho_{2}\right):=$ $\widetilde{D}_{Q}\left(\rho_{2}, \bar{\theta}\right)$. In contrast to the multi-model approach, this optimization involves for each function evaluation the solution of a global optimization problem for worst-case determination on a continuous domain $\Pi$ (instead of a finite set $\Pi_{N}$ ). This is the same as the validation problem mentioned in the previous paragraph. Therefore, for many parameters, the computational effort can be tremendous and can only be alleviated by using parallel computation techniques.

\section{ILLUSTRATIVE EXAMPLE}

We consider the robust actuator fault detection example of [14] which has been studied also in [7]. Although linear, this example allows us to compare the results with those of [7]. The additive fault system has a standard state space realization (3) with

$$
\begin{gathered}
A(\rho)=\left[\begin{array}{ccc}
-0.8 & 0 & 0 \\
0 & -0.5\left(1+\pi_{1}\right) & 0.6\left(1+\pi_{2}\right) \\
0 & -0.6\left(1+\pi_{2}\right) & -0.5\left(1+\pi_{1}\right)
\end{array}\right] \\
B_{u}=\left[\begin{array}{ll}
1 & 1 \\
1 & 0 \\
0 & 1
\end{array}\right], B_{d}=0, B_{f}=\left[\begin{array}{ll}
1 & 1 \\
1 & 0 \\
0 & 1
\end{array}\right], C=\left[\begin{array}{lll}
0 & 1 & 1 \\
1 & 1 & 0
\end{array}\right] \\
D_{u}=0, D_{d}=0, D_{f}=0 .
\end{gathered}
$$

where $\rho=\left[\begin{array}{ll}\pi_{1} & \pi_{2}\end{array}\right]$. In the expression of $A(\rho), \pi_{1} \in$ $[-0.25,0.25]$ and $\pi_{2} \in[-0.25,0.25]$ are uncertainties in the real and imaginary parts of the two complex conjugated eigenvalues $\lambda_{1,2}=-0.5 \pm j 0.6$ of the nominal state matrix $A(0)$. The fault detector filter is aimed to provide robust fault detection of actuator faults in the presence of these parametric uncertainties.

For the robust synthesis we consider the two cases, when either all parameters are measurable or non measurable. For each case, we used a uniform grid $\Pi_{N}$ for both $\pi_{1}$ and $\pi_{2}$ in the range $[-0.25,0.25]$, with $N=5 \times 5$ values. Monte-Carlo simulations have been performed to validate the achieved designs.

For each of the $N$ models we computed a first order scalar output detector. Each detector has the least achievable order, having a single eigenvalue in -10 . The $i$-th detector has been determined such that the corresponding $1 \times 2$ TFM $R_{f}\left(s, \rho^{(i)}\right)$, has the least DC-gain equal to one. $Q\left(s, \rho^{(i)}\right)$ has a state-space realization $\left(A_{Q}, B_{Q}\left(\rho^{(i)}\right), C_{Q}, D_{Q}\left(\rho^{(i)}\right)\right)$ with $A_{Q}=-10, C_{Q}=1$, and $B_{Q}\left(\rho^{(i)}\right)$ and $D_{Q}\left(\rho^{(i)}\right)$ depending on $\rho^{(i)}$.

The step responses of the family of residual generation systems (of the form (6)) from the faults and control inputs on the chosen uniform grid is shown in Figure 1. As it can be observed, a perfect decoupling is achieved for all parameter values from the control inputs in each grid point. Also the 
designed residual generators have been normalized such that the smallest DC-gain of the transfer functions from the faults to the residual is equal to one (in this case from the fault $f_{2}$ to the residual $r$ ).

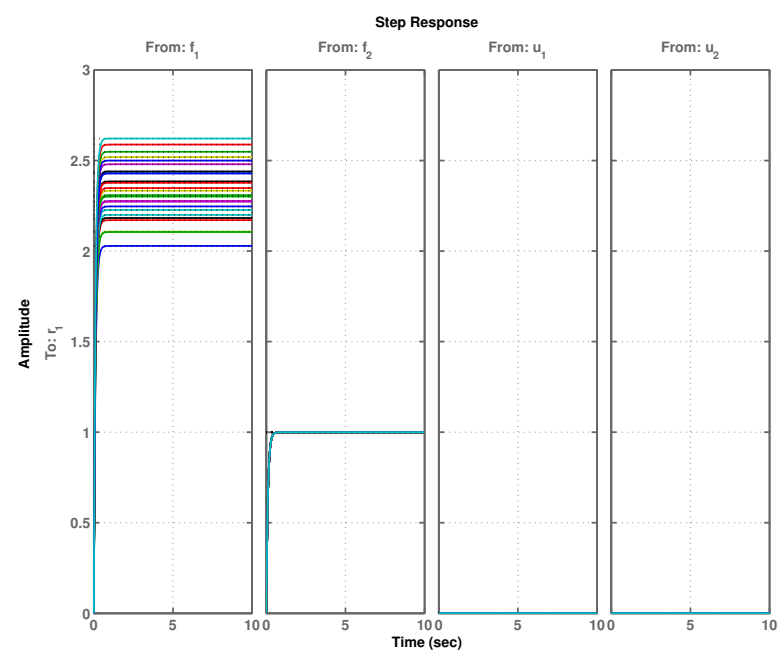

Fig. 1. Step responses of the detection system over the parameter grid

\section{A. Case 1: Both $\pi_{1}$ and $\pi_{2}$ measurable}

Since the continuity condition of the detector state-space matrices with respect to the scheduling parameters can be easily fulfilled for this example, a bilinear interpolation based (2-D look-up table) technique can be employed for the matrices $B_{Q}(\rho)$ and $D_{Q}(\rho)$ to implement the fault detection filter. The Monte Carlo analysis of step responses with the linear interpolation based gain scheduling shows practically no qualitative and quantitative differences to the plots presented in Figure 1. Even a piecewise constant gain scheduling based on switching to the nearest detector provides acceptable results as can be seen in the Monte-Carlo analysis of step responses shown in Figure 2.

As an alternative to using look-up tables, we can determine multivariate polynomial or rational approximations of $B_{Q}(\rho)$ and $D_{Q}(\rho)$ for fixed $A_{Q}$ and $C_{Q}$ matrices (for the exact symbolic expressions see [7]). The main advantage of this approach is that only the continuity of the TFM $Q(s, \rho)$ is required, but not of the state space matrices of its realization. For $\widetilde{B}_{Q}(\rho, \theta)$ and $\widetilde{D}_{Q}(\rho, \theta)$ we employed the affine parameterizations

$$
\begin{aligned}
& \widetilde{B}_{Q}(\rho, \theta)=B_{Q, 0}+\pi_{1} B_{Q, 1}+\pi_{2} B_{Q, 2} \\
& \widetilde{D}_{Q}(\rho, \theta)=D_{Q, 0}+\pi_{1} D_{Q, 1}+\pi_{2} D_{Q, 2}
\end{aligned}
$$

which involve the determination of the best fit of $\theta$ consisting of the 24 unknown entries of the matrices $B_{Q, 0}, B_{Q, 1}, \ldots$, $D_{Q, 2}$. The resulting matrices obtained via optimization are

$$
\left[\begin{array}{l}
\frac{B_{Q, 0}}{B_{Q, 1}} \\
B_{Q, 2}
\end{array}\right]=\left[\begin{array}{rrrr}
24.1051 & -121.6395 & -23.1019 & -9.8865 \\
\hline-8.1627 & 1.9931 & -4.3128 & 0.2891 \\
\hline-13.4739 & -3.1975 & -18.4330 & -13.4203
\end{array}\right]
$$

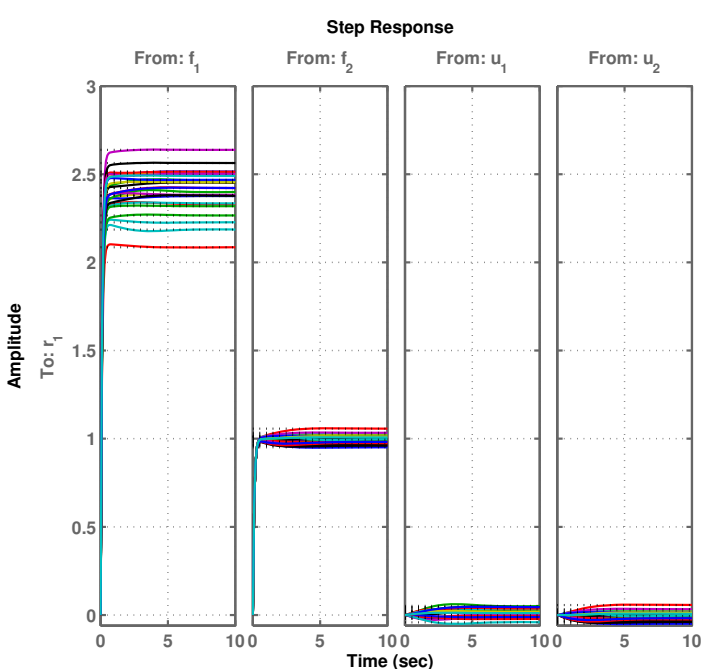

Fig. 2. Step responses of the detection system for piecewise constant gain scheduling

$\left[\begin{array}{l}D_{Q, 0} \\ \hline D_{Q, 1} \\ \hline D_{Q, 2}\end{array}\right]=\left[\begin{array}{rrrr}-3.2035 & 13.2412 & 0.0113 & -0.0002 \\ \hline 0.3396 & 0.4853 & 0.0912 & 0.1104 \\ \hline 0.7432 & 0.7990 & 0.9456 & 0.6408\end{array}\right]$

The minimum achieved worst case value $\max _{\rho \in \Pi_{N}}\left\|(Q(s, \rho)-\bar{Q}(s, \rho)) G_{e}(s, \rho)\right\| \quad$ was 0.3379 for $\pi_{1}=0.25$ and $\pi_{2}=0.25$. This is also the value of the "exact" worst-case value (15) computed by "exact" min-max optimization, relying on the computation of $Q(s, \rho)$ "on-the fly".

In Figure 3 we present the results of parametric analysis of step responses for the affine LPV detector. As it can be observed, a satisfactory approximate decoupling is achieved for all parameter values from the control inputs. Thus, the detection of constant faults (e.g., abrupt changes) can be reliably performed in the presence of measurable parametric uncertainties.

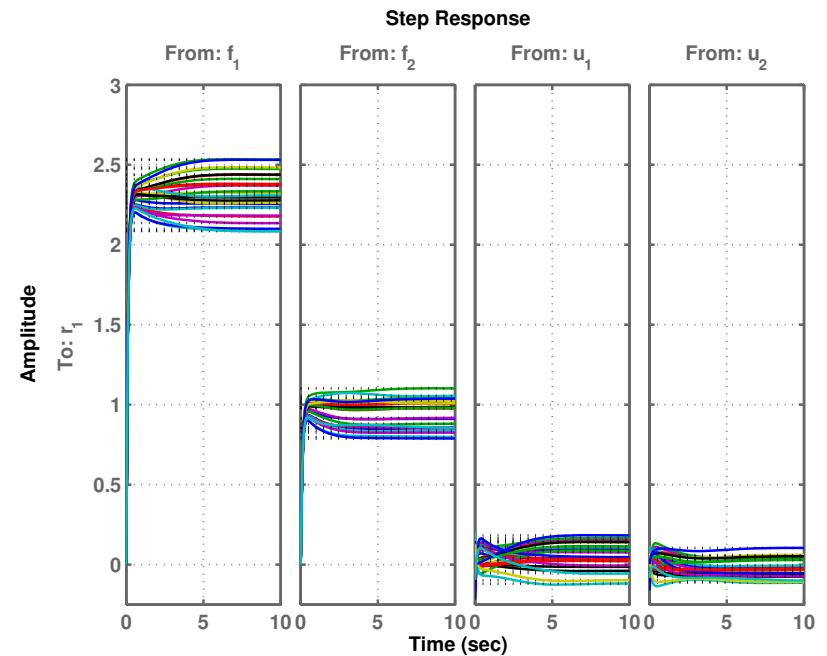

Fig. 3. Parametric step response analysis of the LPV detector robustness 


\section{B. Case 2: Both $\pi_{1}$ and $\pi_{2}$ not measurable}

We determined the best approximations of $B_{Q}(\rho)$ and $D_{Q}(\rho)$ with constant matrices $\bar{B}_{Q}$ and $\bar{D}_{Q}$ over a grid of $N=25$ values. The resulting matrices are

$$
\begin{gathered}
\bar{B}_{Q}=\left[\begin{array}{llll}
24.9716 & -122.0728 & -23.5364 & -10.0792
\end{array}\right] \\
\bar{D}_{Q}=\left[\begin{array}{llll}
-3.2114 & 13.2408 & 0.0008 & -0.0004
\end{array}\right]
\end{gathered}
$$

which agree up too three decimal digits with the results computed in [7]. The minimum worst case value $\max _{\rho \in \Pi_{N}}\left\|(Q(s, \rho)-\bar{Q}(s)) G_{e}(s, \rho)\right\|$ was 0.6426 for $\pi_{1}=$ 0.25 and $\pi_{2}=0.25$. This is also the value of the "exact" worst-case value (15) computed by "exact" min-max optimization, relying on the computation of $Q(s, \rho)$ "on-the fly".

In Figure 4 we present the results of parametric analysis of step responses of the parameter dependent residual generation system. As it can be observed, with an appropriate choice of the detection threshold, the detection of constant faults can be reliably performed in the presence of parametric uncertainties.

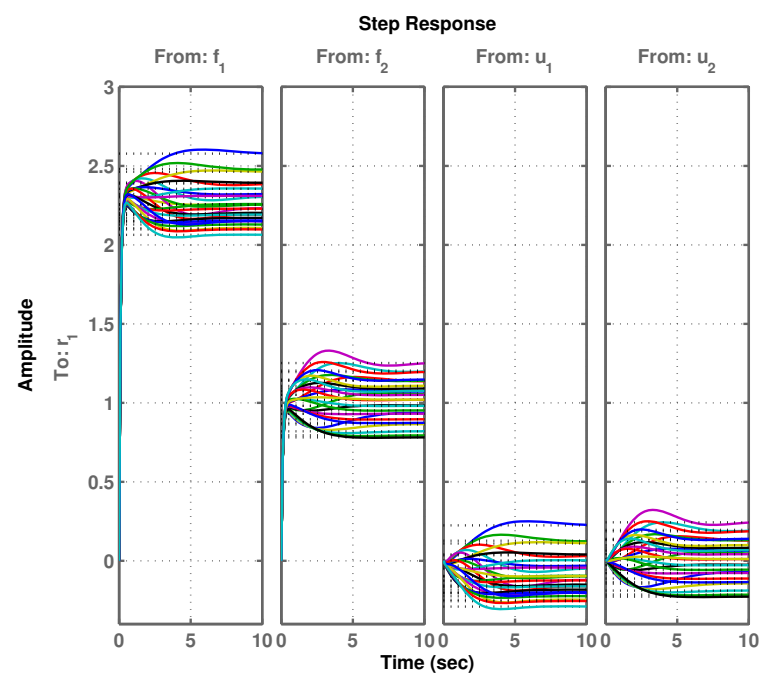

Fig. 4. Parametric step response analysis of constant fitted detector robustness for uncertainties in the real and imaginary parts of the complex conjugated eigenvalues

\section{CONCLUSIONS}

We proposed a robust nullspace synthesis method of LPVscheduling based residual generators. Robustness against parametric uncertainties is provided by a worst-case search based tuning of the LPV-scheduling residual generator. The proposed synthesis method can be also employed for the parametric solution of the fault detection and isolation (FDI) problem using a bank of scalar detectors which provide a set of structured residuals. In combination with trimming and linearization tools, the synthesis method can be also employed to solve fault detection problems for nonlinear systems.

In contrast to the approach proposed in [7] relying on symbolic computational tools, the new synthesis method relies exclusively on numerical computational tools. The basic requirement for the proposed method is the availability of synthesis methods and software tools which guarantee the continuity of the mapping from the uncertain parameters to the resulting transfer-function matrix of the fault detection filter. This requirement can be fulfilled using recently developed software tools for the synthesis of residual generators [2].

Alternative techniques to address the fitting of the detector parameters can be also considered, as for example solving parameter dependent linear matrix inequalities using convex relaxations techniques [15] or using randomization methods to solve semi-infinite optimization problems [16].

\section{ACKNOWLEDGMENTS}

This work was performed in the framework of the ADDSAFE Project: Grant agreement no.: FP7-233815.

\section{REFERENCES}

[1] W. J. Rugh and J. S. Shamma, "Research on gain scheduling," Automatica, vol. 36, no. 10, pp. $1401-1425,2000$.

[2] A. Varga, "Linear FDI-Techniques and Software Tools," German Aerospace Center (DLR), Institute of Robotics and Mechatronics," FAult Detection Toolbox V1.0 - Technical Documentation, 2011.

[3] A. Varga, G. Looye, D. Moormann, and G. Grübel, "Automated generation of LFT-based parametric uncertainty descriptions from generic aircraft models," Mathematical and Computer Modelling of Dynamical Systems, vol. 4, pp. 249-274, 1998.

[4] H. Pfifer and S. Hecker, "Generation of optimal linear parametric models for LFT-based robust stability analysis and control design," Proc. IEEE CDC'09, Cancun, Mexico, 2008.

[5] G. J. Balas, J. Bokor, and Z. Szabó, "Invariant subspaces for LPV systems and their application," IEEE Trans. Automat. Control, vol. 48, pp. 2065-2069, 2003.

[6] J. Bokor and G. Balas, "Detection filter design for LPV systems a geometric approach," Automatica, vol. 40, pp. 511-518, 2004.

[7] A. Varga, "On parametric solution of fault detection problems," Proc. IFAC 2011 World Congress, Milano, Italy, 2011.

[8] E. Frisk and M. Nyberg, "A minimal polynomial basis solution to residual generation for fault diagnosis in linear systems," Automatica, vol. 37, pp. 1417-1424, 2001.

[9] A. Varga, "On designing least order residual generators for fault detection and isolation," Proc. 16th Internat. Conf. on Control Systems and Computer Science, Bucharest, Romania, 2007, pp. 323-330.

[10] M. Kinnaert, R. Hanus, and P. Arte, "Fault detection and isolation for unstable systems," IEEE Trans. Automat. Control, vol. 40, pp. 740$742,1995$.

[11] M. Nyberg, "Criterions for detectability and strong detectability of faults in linear systems," Int. J. Control, vol. 75, pp. 490-501, 2002.

[12] G. Vinnicombe, "Frequency domain uncertainty and the graph topology," IEEE Trans. Automat. Control, vol. 38, pp. 1371-1383, 1993.

[13] A. Varga, "On computing nullspace bases - a fault detection perspective," Proc. IFAC 2008 World Congress, Seoul, Korea., 2008, pp. 6295-6300.

[14] A. Edelmayer and J. Bokor, "Optimal $\mathcal{H}_{\infty}$ scaling for sensitivity optimization of detection filters," Int. J. Robust Nonlinear Control, vol. 12, pp. 749-760, 2002.

[15] C. Scherer, "LMI relaxations in robust control," European Journal of Control, vol. 12, pp. 3-29, 2006.

[16] R. Tempo, G. Calafiore, and F. Dabbene, Algorithms for Analysis and Control of Uncertain Systems. Springer-Verlag, New York, 2004. 